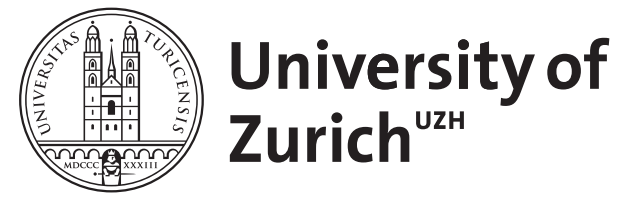

\title{
Analgetikaunverträglichkeit: Intoleranz oder Allergie?
}

Ballmer-Weber, Barbara K

DOI: https://doi.org/10.1024/0040-5930/a001053

Posted at the Zurich Open Repository and Archive, University of Zurich ZORA URL: https://doi.org/10.5167/uzh-177249

Journal Article

Accepted Version

Originally published at:

Ballmer-Weber, Barbara K (2019). Analgetikaunverträglichkeit: Intoleranz oder Allergie? Therapeutische Umschau. Revue thérapeutique, 76(1):23-27.

DOI: https://doi.org/10.1024/0040-5930/a001053 


\title{
Analgetikaunverträglichkeit: Intoleranz oder Allergie?
}

\author{
Barbara K. Ballmer-Weber
}

Fachbereich Allergologie, Klinik für Dermatologie und Allergologie, Kantonsspital St. Gallen

\section{Zusammenfassung:}

Nichtsteroidale Analgetika (NSAID) können sowohl nicht immunologisch vermittelte Intoleranzreaktionen als auch allergische Reaktionen auslösen. Die Intoleranzreaktionen sind häufiger. Klinisch lassen sich Intoleranzreaktionen nicht von allergischen Reaktionen vom Soforttyp unterscheiden. Sie äussern sich hauptsächlich mit kutanen und respiratorischen Symptomen. Da der Wirkmechanismus der nicht-selektiven NSAID, nämlich die Hemmung der Cylclooxygease (COX), v.a. der COX-1, auch die Ursache für die Intoleranzreaktion auf NSAID ist, ist bei vielen Patienten eine Unverträglichkeit auf verschiedene strukturell nicht verwandte COX-1-Hemmer zu befürchten (breite NSAIDIntoleranz). Bei der Allergie auf ein NSAID ist jedoch nur eine Kreuzreaktion zu strukturverwandten NSAID zu erwarten. Wichtig für den Patienten ist es, im Rahmen der allergologischen Abklärung für ihn gut verträgliche Ausweichanalgetika zu identifizieren.

\section{Summary: Hypersensitivity to Analgesics: intolerance or allergy?}

Nonsteroidal anti-inflammatory drugs (NSAID) can induce not immunologically mediated intolerance reaction and allergic reactions. Intolerance reactions are more prevalent than allergic reactions. The clinical manifestation does not differentiate between an intolerance reaction and an immediate type allergy. They usually induce either cutaneous and/or respiratory symptoms. The pathogenesis of NSAID intolerance is based on the inhibition of the cyloclooxygenase (COX), particularly the COX-1, which is however the mechanism of action on which NSAIDs exert their intended effects. Therefore, cross-reactivity to many structurally nonrelated NSAIDs has to be expected (broad NSAID intolerance). In case of an allergy to a NSAID, however, only cross-reactivity to structurally related drugs occurs. It is very important for the patient with intolerance reactions to NSAIDs to identify well tolerated alternative analgesics. 


\section{Einleitung}

Dem Chemiker Felix Hoffmann gelang es 1897 Acetylsalicylsäure in einer chemisch stabilen und reinen Form zu produzieren. 1899 wurde Acetylsalicylsäure unter dem Namen Aspirin patentrechtlich geschützt und durch die Firma Bayer vermarktet. Weltweit werden ca. 50`000 Tonnen Aspirin pro Jahr konsumiert. Die erste Unverträglichkeitsreaktion auf Aspirin wurde bereits 1902 publiziert. Ein Patient entwickelte ein periorbitales Angioödem und eine Urtikaria drei Stunden nach Einnahme des Medikamentes. Widal und Mitarbeiter beschrieben 1922 das „Aspirin-idiosyncrasy-asthma-nasal polyposis-syndrome (plus urticaria)“. Max Samter wies in seiner Publikation 1967 auf das häufige Auftreten von Angioödem, Rhinitis und Asthma bei Patienten unter Aspirin-Therapie hin und erörterte, dass es sich dabei nicht um eine Allergie handle. Er beschrieb ca. 1000 Patienten mit diesem Symptomenkomplex. Die Erkrankung wurde in der Folge über viele Jahre als Samter-Trias, Morbus Samter oder Morbus Widal benannt [1].

\section{Einteilung der nicht-opioiden Schmerzmittel}

Nicht-opioide Schmerzmittel gehören zu den weltweit am häufigsten eingenommenen und auch verordneten Medikamenten. Sie haben nicht nur eine schmerzlindernde (analgetische) Wirkung, sondern teilweise auch einen fiebersenkenden (antipyretischen) und entzündungshemmenden (antiphlogistischen) Effekt. Sie werden eingeteilt in die Gruppe der nicht-selektiven NSAID (englisch non-steroidal anti-inflammatory drugs, nichtsteroidale Analgetika), deren Vertreter eine analgtische, antipyretische und antiphlogistische Wirkung haben und die aus den Untergruppen der sauren, antipyretisch antiphlogistischen Analgetika (z.B. Aspirin) sowie der selektiven COX-2-Hemmern (z.B. Etoricoxib) besteht, und in die Gruppe der nichtsauren antipyretischen Analgetika (Paracetamol, Metamizol), welche eine analgetische und antipyretische Wirkung haben. Ihnen allen gemeinsam ist die Hemmung der Cyclooxygenase. Der Einfachheit halber werden diese Substanzen in der Folge unter dem Überbegriff der NSAID zusammengefasst. Tabelle 1 gibt einen Überblick zur Einteilung der NSAID gemäss ihren chemischen Strukturen [2].

\section{Die Pathogenese der NSAID-Intoleranz}

Die Pathogenese der NSAID-Intoleranz ist bis heute nicht definitiv geklärt. Die nicht selektiven NSAID hemmen beide Isoformen der Cyclooxygenase (COX-1 und COX-2), wobei diese Hemmung zentral ist für die analgetische, antipyretische und antiphlogistische Wirkung dieser Medikamente.

Die Arachidonsäure wird v.a. durch die Cylcooxygenase-1 zu protektiven Prostaglandinen abgebaut, während die Lipoxygenase die Arachidonsäure zu proinflammatorischen Leukotrienen metabolisiert (Abb 1). V.a. NSAID mit starker COX-1-Hemmung können bei 
genetisch prädisponierten Patienten zu einem Ungleichgewicht führen zwischen Prostaglandinen und Leuktotrienen und so u.a. eine Bronchokonstriktion wie auch weitere Beschwerden induzieren [3]. Schwache COX-1 Hemmer und v.a. COX-2 Hemmer werden von Patienten mit einer NSAID-Intoleranz häufig (aber nicht immer!) gut toleriert, da letztere die COX-1 in ihrer Funktion wenig oder nicht beeinflussen. Tabelle 2 zeigt die Klassifikation der NSAID entsprechend ihrer COX-1-Hemmung [4-5]. Zu erwähnen ist, dass die Inoleranzreaktion dosisabhängig ist. Zum Beispiel ist Paracetamol zwar nur ein schwacher COX-1 Hemmer, kann in hohen Dosen aber trotzdem bei einem Teil der Patienten mit einer NSAID-Intoleranz zu Beschwerden führen. Da der Wirkmechanismus der nicht-selektiven NSAID, nämlich die Hemmung der COX-1 auch die Ursache für die Intoleranzreaktion auf NSAID ist, ist bei vielen Patienten eine Unverträglichkeit auf die meisten COX-1-Hemmer zu befürchten (sogenannte breite NSAID-Intoleranz).

Es gibt Patienten, die auf NSAID eine „echte“ Allergie entwickeln. Diese kann sich als Soforttypallergie manifestieren und führt hauptsächlich zu kutanen (Urtikaria, Angioödme), teils aber auch respiratorischen Beschwerden bis zur Anaphylaxie. Andererseits werden auch seltener Spättypallergien (T-Zell-vermittelt) beobachtet, welche zu Arzneimittelexanthemen führen. Bei Patienten mit einer „echten“ Allergie ist in der Regel nur eine einzige Substanzgruppe (auslösendes Medikament und dazu strukturverwandte Medikamente) betroffen.

Um die Sache zu komplizieren, werden Patienten beobachtet, die nur auf ein NSAID reagieren (z.B. Iburprofen, Diclofenac, Aspirin, Paracetamol oder Pyrazolone [2]), bei denen jedoch eine „echte“ Allergie diagnostisch nicht erfasst werden kann. Die Pathogenese ist hier nicht geklärt.

\section{Das klinische Erscheinungsbild}

Der Begriff NSAID-Intoleranz oder NSAID-Hypersensitivität bezog sich ursprünglich auf die von Widal oder Samter beschriebene Symptomen-Trias. Das klinische Bild der von Samter und Widal beschriebenen Erkrankung ist relativ gut definiert. Der Beginn liegt meistens im dritten Lebensjahrzehnt und setzt mit einer chronischen Rhinitis ein, welche häufig nach einem viralen Infekt des Respirationstraktes beginnt. Häufig finden sich bereits in dieser Phase nasale Polypen. Über Monate bis Jahre entwickelt sich ein Asthma bronchiale und eine NSAID-Intoleranz. Die Einnahme von NSAID führt bei diesen Patienten nach einer bis mehreren Stunde häufig zu einer bronchialen Obstruktion, Rhinitis, konjunktivaler Injektion, sowie zu einem Flush von Gesicht und Nacken. Trotz Vermeiden der NSAID persistieren die Polyposis nasi und das Asthma bronchiale. Häufig findet sich eine Blut-Eosinophilie sowie eine Gewebe-Eosinophilie der nasalen und bronchialen Mukosa. 
Da sich NSAID-Unverträglichkeiten aber auch ausserhalb dieser von Widal und Samter beschriebenen eosinophilen Erkrankung der Atemwege finden und wie auch von beiden Autoren beschrieben neben respiratorischen Symptomen auch zu Urtikaria und Angioödemen führen können, sowie pathogenetisch nicht nur aufgrund einer Intoleranzreaktion, sondern auch aufgrund einer „echten“ Allergie entstehen können, wurde in der neuen Literatur, u.a. durch die EAACI (European Academy of Allergy and Clinical Immunology) eine Einteilung vorgeschlagen, die auf fünf klinischen Entitäten der Unverträglichkeitsreaktionen auf NSAID beruht $[2,6]$. Dabei werden die NSAID-IntoleranzReaktionen, die nicht immunologisch bedingt sind und auf einer Störung im ArachidonsäureMetabolismus beruhen, von echten allergischen Reaktionen (Allergie vom Soforttyp und Allergie vom Spättyp) abgegrenzt. Aufgrund des klinischen Erscheinungsbildes kann eine Intoleranzreaktion von einer Allergie nicht unterschieden werden.

Gemäss der neuen Einteilung werden folgende fünf Untergruppen unterschieden, die in Tabelle 3 zusammengefasst sind. Zu erwähnen ist, dass Intoleranzreaktionen (1-3) viel häufiger auftreten als echte allergische Reaktionen (4-5).

1. Patienten mit chronisch spontaner Urtikaria oder Angioödeme, die nach Einnahme von NSAID aufgrund einer Intoleranz eine Exazerbation ihrer Grundkrankheit erfahren

2. Patienten ohne Grunderkrankung, die nach Einnahme von NSAID aufgrund einer Intoleranz eine Urtikaria oder ein Angioödem entwickeln

3. Patienten mit einem Asthma bronchiale und/oder einer Polyposis nasi (entsprechend der ursprünglichen Widal/Samter Trias), die aufgrund einer Intoleranz eine Exazerbation ihrer respiratorischen Erkrankung erfahren

4. Patienten, die aufgrund einer IgE-vermittelten Allergie eine Allergie vom Soforttyp erfahren.

5. Patienten, die aufgrund einer T-Zell-vermittelten Allergie eine Allergie vom Spättyp erfahren. Diese kann sich als banales makulopapulöses Exanthem bis zur schweren Arzneimittelreaktion, wie z.B. toxisch epidermale Nekrolyse (TEN), Steven-Johnson-Syndrom, akute generalisierte exanthematische Pustulose (AGEP) oder einer „Drug reaction with eosinophila and systemic symptoms (DRESS)“ manifestieren.

\section{Die Abklärung bei Patienten mit einer Unverträglichkeitsreaktion auf NSAID}

Wichtig bei der Diagnostik von Unverträglichkeitsreaktionen auf NSAID ist die Anamnese [7]. Sie kann bereits richtungsweisend sein. Insbesondere relevant ist die Frage nach dem Vorliegen von Grunderkrankungen, wie Asthma bronchiale, nasale Polypen oder einer 
chronisch spontanen Urtikaria/Angioödme. Ebenso ist die anamnestische Angabe, ob andere NSAID toleriert werden oder nicht, wichtig.

Mittels Hauttestung wird in einem nächsten Schritt durch den Allergologen eine „echte“ Allergie ausgeschlossen rsp. nachgewiesen (Abb. 2). In Einzelfällen können zusätzliche Labortests hilfreich sein: Der Basophilenaktivierungstest (BAT) zum Ausschluss/Nachweis der Soforttypallergie oder der Lymphozytentransformationstest (LTT) bei Verdacht auf eine Spättypallergie (siehe Beitrag Hausmann und Müller in diesem Themenheft).

Das oberste Ziel der Abklärung ist die Austestung von gut verträglichen Alternativmedikamenten, welche unter kontrollierten, titrierten, meist oralen Provokationen und meist im klinischen Setting erfolgt. Bei Patienten mit der Anamnese einer breiten NSAIDIntoleranz werden Provokationen mit schwachen COX-1-Hemmern und v.a. auch mit COX-2Hemmern durchgeführt. Da auch diese Substanzgruppen zu Unverträglichkeitsreaktionen führen können, ist eine kontrollierte Provokation unerlässlich.

Besteht der Verdacht auf eine Intoleranz auf ein einzelnes NSAID, wird in der Regel im klinischen Setting eine Provokation mit Aspirin in hohen Dosen durchgeführt. Wenn der Patient diese hochdosierte Provokation gut toleriert, kann eine breite NSAID-Intoleranz ausgeschlossen werden. In der Folge können alternative NSAID ausgetestet werden. Wichtig ist die Abgabe eines Allergiepasses, in dem einerseits die unverträglichen NSAID notiert werden, andererseits aber auch die vom Patienten gut tolerierten Analgetika festgehalten werden.

\section{Therapeutische Ansätze}

Die NSAID-Intoleranz kann nicht ursächlich therapiert werden. Es hat sich jedoch gezeigt, dass mit langsamer Dosissteigerung beginnend mit kleinsten Mengen an Aspirin eine Gewöhnung an das Medikament bei einem Teil der Patienten erzielt werden kann. Dieses Vorgehen der Toleranzinduktion wird auch adaptive Desaktivierung genannt. Die Toleranz wird jedoch nur aufrechterhalten, wenn der Patient das Medikament täglich einnimmt und wird nach 2-5 Tagen nach Therapiestopp durchbrochen. Die Indikation für eine adaptive Desaktivierung mit Aspirin ist v.a. bei Patienten mit breiter NSAID-Intoleranz rsp einer Aspirin-Unverträglichkeit und Indikation für eine niedrigdosierte Aspirin-Therapie gegeben. Hier wird durch vorsichtige Dosissteigerung im klinischen Setting unter optimaler Monitorisierung der Vitalparameter eine Dosis von 100mg Aspirin in mehreren Schritten angestrebt.

Bei Patienten mit einer Samter- oder Widal Trias, rsp einer „NSAID-exacerbated respiratory disease (NERD)“ kann eine adaptive Desaktivierung bis zu 1.3g Aspirin veruscht werden mit dem Ziel sowohl die Asthma-Exazerbationen also auch die Rezidive der nasalen Polyposis zu durchbrechen [1]. Bei erfolgreichem Ansprechen wird die Dosis auf ca 600mg pro Tag 
gesenkt. Aufgrund der häufigen Nebenwirkungen wird diese Therapieform kontrovers beurteilt [7].

\section{Diätische Implikationen bei Aspirin-Unverträglichkeit?}

Acetylsalicylsäure kommt in der Natur nicht vor, jedoch ihre Ausgangssubstanz, die sogenannte Salicylsäure oder das Salicylat, welche ausser in alkoholischen Getränken, in Früchten, Gemüsen, Kräutern und Gewürzen zu finden sind. Eine Evidenz für eine Relevanz dieser in Lebensmittel enthaltenen Salicylate auf den Verlauf einer Aspirin-Unverträglichkeit rps einer „NSAID-exacerbated respiratory disease (NERD)“ ist nicht gegeben. Mit einer normalen Diät werden pro Tag ca 3-5mg Salicylat eingenommen. Diese Dosis liegt weit unter der Aspirindosis, welche zu Beschwerden führen kann und hat keine direkte Wirkung auf die COX-1 und COX-2. Die meisten Patienten mit Aspirin-Intoleranz entwickeln nach Einnahme von ca 100mg Aspirin Beschwerden. Umgerechnet auf die Dosis an Salicylaten in Lebensmitteln würde dies einer Menge von $20 \mathrm{~kg}$ Kirschen oder $3 \mathrm{~kg}$ Rosinen entsprechen [8]. Aufgrund dieser Tatsachen ist eine Salicylatreduktion in der Nahrungs zur Therapie der Aspirin- rsp. NSAID-Unverträglichkeit weder pathogenetisch begründbar noch zu empfehlen. Diese Sachverhalte wurden in einer Stellungsnahme der deutschsprachigen Arbeitsgemeinschaft für Nahrungsmittelallergie kürzlich zusammengefasst (Artikel in press). 


\section{Literatur}

1. White AA, Stevenson DD. Aspirin-Exacerbated Respiratory Disease. N Engl J Med 2018;379:1060-70.

2. Kowalski ML, Asero R, Bavbek S et al. Classification and practical approach to the diagnosis and management of hypersensitivity to nonsteroidal anti-inflammatory drugs. Allergy 2013;68:1219-32.

3. Wedi B. Aktuelle Diagnostik der NSAR-Überempfindlichkeit. Allergo J 2017;26:20411.

4. Warner TD, Mitchell JA. Cyclooxygenases: new forms, new inhibitors, and lessons from the clinic. FASEB J 2004;18:790-804.

5. Thong BY. Nonsteroidal anti-inflammatory drug hypersensitivity in the Asia-Pacific. Asia Pac Allergy 2018;23:e38.

6. Wöhrl S. NSAID hypersensitivity - recommendations for diagnostic work up and patient management. Allergo J Int 2018;27:114-21.

7. Kowalski ML, Agache I, Bavbek S et al. Diagnosis and management of NSAIDExacerbated Respiratory Disease (N-ERD)-a EAACI position paper. Allergy. 2019;74:28-39.

8. Plank-Habibi S, Dölle S, Schäfer C. Diätische Implikationen: Salicylsäure und ASSUnverträglichkeit. Allergologie 2018;41:261-272.

Korrespondenzadresse:

Prof. Dr. med. Barbara K. Ballmer-Weber

Fachbereich Allergologie

Klinik für Dermatologie und Allergologie

Kantonsspital St. Gallen

Rorschacherstr. 95

9007 St. Gallen

Email:barbara.ballmer-weber@kssg.ch

Tel: 071'4947340 
Abb 1

\section{Phospholipid}

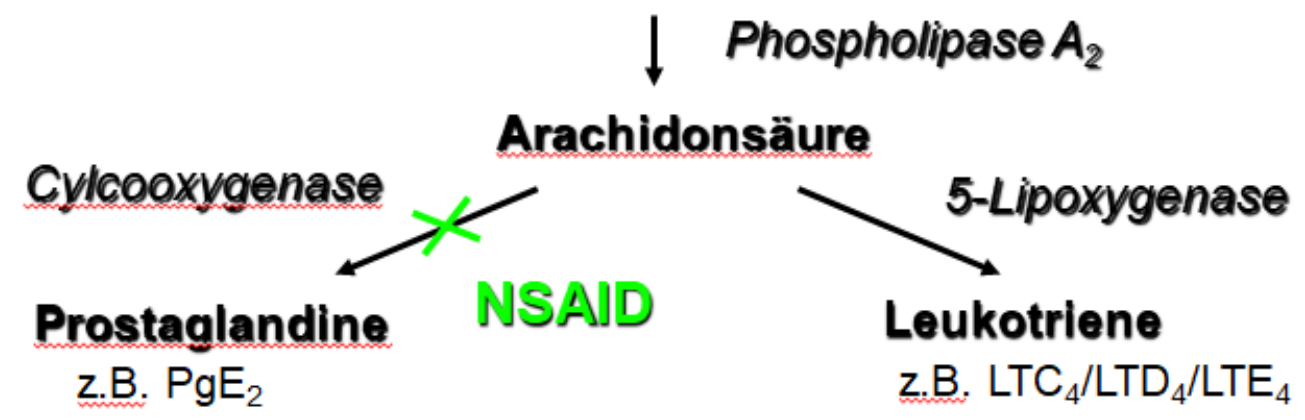

Abb 1: Indem die NSAID die Cyclooxygenase (COX), v.a. die COX-1 hemmen, kommt es zu einem Ungleichgewicht im Arachidonsäuremetabolismus und bei genetisch prädisponierten Patienten zu einer Intoleranzreaktion.

Abb 2

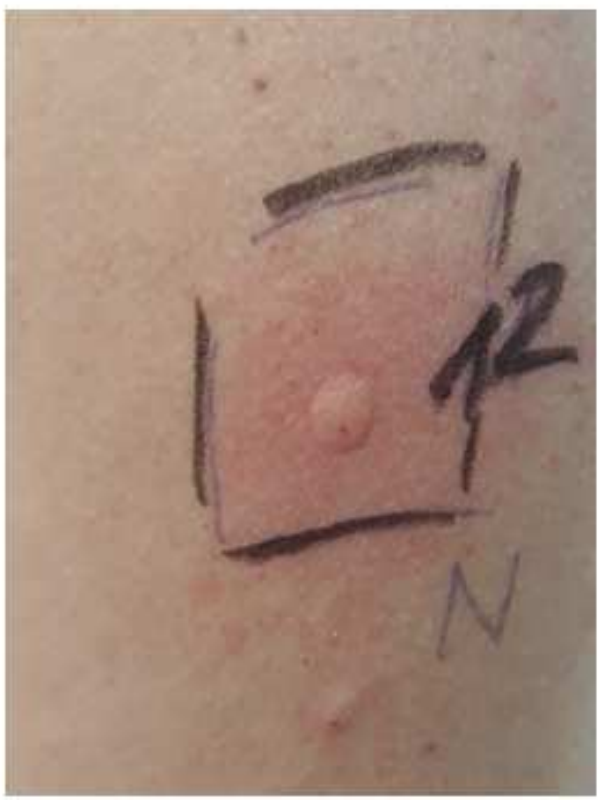

Abb 2: Patientin mit Urtikaria und Bronchospasmus 15 Minuten nach Einnahme von Metamizol. Die positive Intrakutantestung war hinweisend für das Vorliegen einer Soforttypallergie auf Metamizol, Parallel dazu verlief auch ein Basophilenaktivierungstest positiv. Für diese Patientin ist Metamizol/alle Pyrazolone gesperrt, alle anderen NSAID stehen ihr zur Verfügung 


\section{Legenden zu den Abbildungen:}

Abb 1: Indem die NSAID die Cyclooxygenase (COX), v.a. die COX-1 hemmen, kommt es zu einem Ungleichgewicht im Arachidonsäuremetabolismus und bei genetisch prädisponierten Patienten zu einer Intoleranzreaktion.

Abb 2: Patientin mit Urtikaria und Bronchospasmus 15 Minuten nach Einnahme von Metamizol. Die positive Intrakutantestung war hinweisend für das Vorliegen einer Soforttypallergie auf Metamizol. Parallel dazu verlief auch ein Basophilenaktivierungstest positiv. Für diese Patientin sind Metamizol rsp. alle Pyrazolone gesperrt, alle anderen NSAID stehen ihr zur Verfügung 
Tabelle 1: Einteilung der NSAID gemäss ihrer chemischen Struktur (angepasst nach [1])

\begin{tabular}{|l|l|}
\hline Chemische Gruppe & Beispiele: Medikamente \\
\hline Salicylsäure Derivate & Aspirin (Acetylsalicylsäure) \\
& Sulfasalazin \\
\hline Para-Aminophenol & Acetaminophen/Paracetamol \\
\hline Propionsäure Derivate & Ibuprofen \\
& Naproxen \\
& Ketoprofen \\
& Flurbiprofen \\
\hline Arylessigsäure Derivate & Diclofenac \\
& Etodolac \\
& Ketolorac \\
& Indomethacin \\
\hline Enolsäure Derivate & Pyrazolone \\
& - Metamizol \\
& Oxicame \\
& - Piroxicam \\
& - Tenoxicam \\
& - Lornoxicam \\
\hline Fenaminsäure Derivate & Mefenaminsäure \\
\hline Selektive COX-2 Hemmer & Celecoxib \\
(Coxibe) & Etoricoxib \\
\hline
\end{tabular}

Tabelle 2: Einteilung der NSAID gemäss ihrer Hemmung der COX-Isoformen (angepasst nach $[1,5]$ )

\begin{tabular}{|c|c|c|c|}
\hline COX-Hemmung & COX-1-Hemmung & COX-2-Hemmung & Medikamente \\
\hline $\begin{array}{c}\text { Starke COX-1- } \\
\text { Hemmung }\end{array}$ & +++ & $\begin{array}{c}\text { Nicht-selektive NSAID } \\
\text { (z.B. Aspirin, lbuprofen, } \\
\text { Mefenaminsäure) }\end{array}$ \\
\hline $\begin{array}{c}\text { Schwache COX-1- } \\
\text { Hemmung }\end{array}$ & Bei hoher Dosis & $(-)$ & Paracetamol \\
\hline $\begin{array}{c}\text { Stärkere COX-2 als } \\
\text { COX-1-Hemmung }\end{array}$ & Bei hoher Dosis & + & Nimesulid, Meloxicam \\
\hline $\begin{array}{c}\text { Selective COX-2- } \\
\text { Hemmung }\end{array}$ & $(-)$ & +++ & Etoricoxib. Celecoxib \\
\hline
\end{tabular}


Table 3: Die fünf Manifestationsformen der NSAID-Unverträglichkeit (angepasst gemäss [2,6])

\begin{tabular}{|l|l|l|l|l|}
\hline $\begin{array}{l}\text { Untergruppen } \\
\text { der NSAID- } \\
\text { Unverträglichkeit }\end{array}$ & Pathogenese & Symtptome & $\begin{array}{l}\text { Zugrundeliegende } \\
\text { Erkrankung }\end{array}$ & $\begin{array}{l}\text { Kreuzreaktionen zu } \\
\text { anderen NSAID zu } \\
\text { erwarten? }\end{array}$ \\
\hline $\begin{array}{l}\text { NSAID-exacerbated } \\
\text { respiratory disease } \\
\text { (NERD) }\end{array}$ & $\begin{array}{l}\text { Cox-1-Hemmung } \\
\text { (Intoleranz) }\end{array}$ & $\begin{array}{l}\text { Respirationstrakt } \\
\text { Asthma, nasale } \\
\text { Kongestion } \\
\text { Rhinorrhoe }\end{array}$ & $\begin{array}{l}\text { Asthma bronchiale } \\
\text { Nasale Polypen }\end{array}$ & ja \\
\hline $\begin{array}{l}\text { NSAID-exacerbated } \\
\text { cutaneous disease } \\
\text { (NECD) }\end{array}$ & $\begin{array}{l}\text { Cox-1-Hemmung } \\
\text { (Intoleranz) }\end{array}$ & $\begin{array}{l}\text { Hautbeschwerden } \\
\text { Quaddeln, } \\
\text { Angioödeme }\end{array}$ & $\begin{array}{l}\text { Chronisch spontane } \\
\text { Urticaria oder } \\
\text { Angioödeme }\end{array}$ & ja \\
\hline $\begin{array}{l}\text { NSAID-induced } \\
\text { urticaria/angioedema } \\
\text { (NIUA) }\end{array}$ & $\begin{array}{l}\text { Cox-1-Hemmung } \\
\text { (Intoleranz) }\end{array}$ & $\begin{array}{l}\text { Quaddeln, } \\
\text { Angioödeme }\end{array}$ & keine & ja \\
\hline $\begin{array}{l}\text { Single NSAID induced } \\
\text { urticaria, angioedema } \\
\text { or anaphylaxis } \\
\text { (SNIUAA) }\end{array}$ & $\begin{array}{l}\text { Allergie vom } \\
\text { Soforttyp möglich } \\
\text { (IgE vermittelt) }\end{array}$ & $\begin{array}{l}\text { Urtikaria, Angioödem, } \\
\text { Bronchospasmus, } \\
\text { Rhinitis, Conjunctivitis, } \\
\text { Blutdruckabfall, Schock }\end{array}$ & keine & $\begin{array}{l}\text { nein } \\
\text { (nur innerhalb der } \\
\text { gleichen chemischen } \\
\text { Gruppe, bei } \\
\text { verwandter Struktur) }\end{array}$ \\
\hline $\begin{array}{l}\text { Single NSAID induced } \\
\text { delayed reaction } \\
\text { (SNIDR) }\end{array}$ & $\begin{array}{l}\text { Allergie vom } \\
\text { Spättyp } \\
\text { (T-Zell vermittelt) }\end{array}$ & $\begin{array}{l}\text { Makulopapulöses } \\
\text { Exanthem, } \\
\text { Kontaktallergie, fixes } \\
\text { toxisches Arzneimittel- } \\
\text { exanthem, schwere } \\
\text { kutane } \\
\text { Arzneimittelreaktion }\end{array}$ & keine & $\begin{array}{l}\text { nein } \\
\text { (nur innerhalb der } \\
\text { gleichen chemischen } \\
\text { Gruppe, bei } \\
\text { verwandter Struktur) }\end{array}$ \\
\hline
\end{tabular}

